\title{
The Effects of Post-Stenotic Dilatations on the Flow of Couple Stress Fluid through Stenosed Arteries
}

\author{
K. Maruthi Prasad, T. Sudha*, M. V. Phanikumari \\ Department of Mathematics, School of Technology, GITAM University, Hyderabad, India \\ Email: *sudhathulluri@gmail.com
}

How to cite this paper: Maruthi Prasad, K. Sudha, T. and Phanikumari, M.V. (2016) The Effects of Post-Stenotic Dilatations on the Flow of Couple Stress Fluid through Stenosed Arteries. American Journal of Computational Mathematics, 6, 365-376. http://dx.doi.org/10.4236/ajcm.2016.64036

Received: November 21, 2016 Accepted: December 26, 2016

Published: December 29, 2016

Copyright $\odot 2016$ by authors and Scientific Research Publishing Inc. This work is licensed under the Creative Commons Attribution International License (CC BY 4.0).

http://creativecommons.org/licenses/by/4.0/ (c) (i) Open Access

\begin{abstract}
The flow of incompressible couple stress fluid in a circular tube with stenosis and dilatations has been investigated. The stenosis was assumed to be axially symmetric and mild. The flow equations have been linearized and the expressions for the resistance to the flow, velocity, pressure drop, wall shear stress have been derived. The effects of various parameters on these flow variables have been investigated. It is found that the resistance to the flow and pressure drop increase with height of the stenosis and decrease with post stenotic dilatation. Pressure drop decreases with couple stress fluid parameter for both stenosis and post stenotic dilatation. Further, the wall shear stress increases with height of the stenosis and couple stress parameter but decreases with post stenotic dilatation and couple stress fluid parameter.
\end{abstract}

\section{Keywords}

Stenosis, Dilatation, Wall Shear Stress, Resistance to the Flow, Couple Stress Fluid Parameter

\section{Introduction}

In the present world, diseases in the blood vessels and in the heart like heart attack and stroke are major health hazards causing a large number of deaths. The main reason for these deaths is stenosis. The actual causes for stenosis are not well known but it has been suggested that deposits of fatty substances, cholesterol, cellular waste products on the arterial wall are called as stenosis. When stenosis is formed in the artery then these fatty substances can block an artery and blood flow will be restricted. This may lead to heart attacks, hypertension and brain strokes etc. Hence formation of the stenosis i.e. abnormal and unnatural growth on the arterial wall can disturb the normal functioning 
of the cardiovascular system and there is considerable evidence that hydro-dynamical factors such as change in pressure, wall shear stress and impedance can play a significant role in the development and progression of this pathological condition. Hence detailed knowledge of the blood flow in stenosed arteries is required to understand and prevent arterial diseases.

Based on this large number of studies, it has been available to understand flow of blood through arteries. The following literatures have been carried out on the assumption that blood behaves like a non-Newtonian fluid. And it is well known that, blood being the suspension of cells, behaves like a non-Newtonian fluid at low shear rates and during its flow through narrow blood vessels. Shukla et al. [1] studied the effects of stenosis on Non-Newtonian flow of the blood in an artery. Chakravarty Santabrata et al. [2] discussed dynamic response of arterial blood flow in the presence of multi-stenoses. Philip et al. [3] studied the flow of blood, and modeled it by a simple micro fluid in the core region with Newtonian fluid peripheral layer, in a tube in the presence of mild stenosis. Prashanth Kumar [4] studied on unsteady analysis of non-Newtonian blood flow through tapered arteries with a stenosis. Sankar and Hemalatha [5] investigated on steady flow of Herschel-Bulkley fluid through catheterized arteries. Maruthi Prasad et al. [6] have developed the flow of Herschel-Bulkley fluid through an inclined tube of nonuniform cross section with multiple stenoses. Effect of paired stenosis on blood flow through small artery was presented by Yaseen Ali khan Mea [7]. Sreenadh et al. [8] developed a model and analyzed flow of a Casson fluid through an inclined tube of non-uniform cross section with multiple stenoses. Rekha Bali et al. [9] developed mathematical model with multiple stenosis in presence of magnetic field by treating blood as Casson fluid. Mathematical analysis of Casson fluid model for blood rheology in stenosed narrow arteries was studied by Venkatesan et al. [10].

Couple stress fluid is a special case of non-Newtonian fluid which was developed by Stokes [11]. The important feature of these fluids is that stress tensor is not symmetric and their accurate flow behavior cannot be predicted by classical Newtonian theory. The main effect of couple stress will introduce a size-dependent effect that is not present in the classical viscous theories. Couple stress fluid model has been widely used by researchers because of its relative mathematical simplicity compared with other models. Blood, lubricants containing small amount of high polymer additives, electro-rheological fluids and synthetic fluids show the effect of couple stress and rotation of molecules, which are not present in the case of Newtonian fluids. Bringing a few on couple stress fluid, Srivastava [12] considered the flow of couple stress fluid through stenotic vessels with peripheral layer. Srinivasacharya et al. [13] investigated on effects of couple stresses on the flow in a constricted annulus. Effect of peripheral layer on peristaltic transport of couple stress fluid was studied by Maruthiprasad et al. [14]. Pulsatile flow of couple stress fluid through a porous medium with periodic body acceleration and magnetic field was presented by Rathod et al. [15]. Srikanth et al. [16] have analyzed the mathematical problems of non-Newtonian fluid through multiple stenosis arteries in the presence of couple stress. Peristaltic transport of a couple stress fluid has 
been discussed by Maiti et al. [17]. Gruju Awgichew et al. [18] described the effect of slip condition on couple stress fluid flow through porous medium with stenosis.

Arterial dilatation distal to a stenosis has been known as post stenotic dilatation. It is observed that, the nervous system in some people is weak (especially in old people). When blood is clotted at particular position, after that position the arterial wall bulges out (due to high pressure). If it goes on increasing, there is chance to harm the arterial walls. It may causes for death. The exact flow disturbances is uncertain, statis, increased lateral pressure, cavitations, abnormal shear stresses and turbulence all have been postulated to be the causes of post stenotic dilatation. Therefore, understanding the dilatation problems will more helpful to diagnoses the arterial diseases. Based on this, Pincombe et al. [19] studied the analysis of post-stenotic dilatations on the blood flow through the stonosed coronary arteries. Singh AK et al. [20] presented the effects of post stenotic dilatation by treating the fluid as Bingham fluid. Sanjeev Kumar et al. [21] described blood flow resistance for a small artery with multiple stenosis and post stenotic dilatation. Maruthi Prasad et al. [22] investigated on post stenotic dilatation by considering blood as Jeffrey fluid.

Motivated from the above studies a mathematical model was developed by considering blood as couple stress fluid, passing through an artery (tube) having stenosis and post stenotic dilatations. The velocity, impedance (resistance to the flow) and wall shear stress are calculated. The variation of impedance and shear stress is analyzed for various values of couple stress fluid parameters and geometric parameters.

\section{Mathematical Formulation}

Consider the flow of incompressible couple stress fluid through an axisymetric artery (tube) containing stenosis and dilatation as shown in Figure 1.

The equations describing the geometry of the wall is given as follows

$$
\begin{cases}1-\frac{\delta_{i}}{2 R_{0}}\left[1+\cos \frac{2 \pi}{L_{i}}\left(Z-\alpha_{i}-\frac{L_{i}}{2}\right)\right] & \text { for } \alpha_{i} \leq Z \leq \beta_{i} \\ 1, & \text { otherwise }\end{cases}
$$

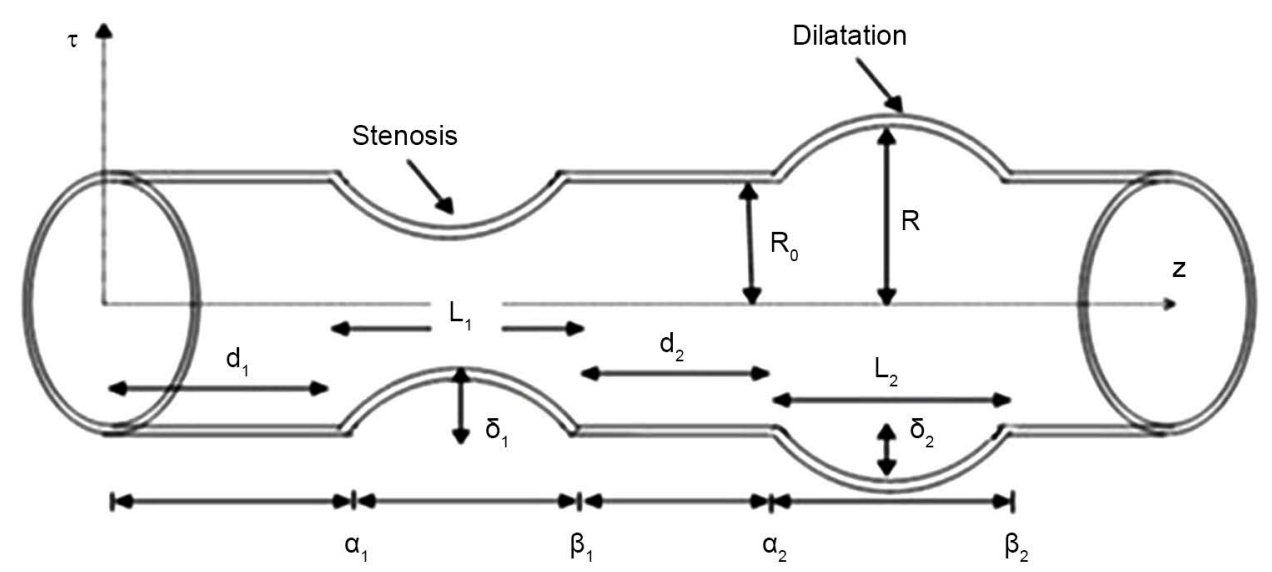

Figure 1. Geometry of arterial segment under consideration. 
where $\delta_{i}$ represents the maximum distance of the $i^{\text {th }}$ abnormal segment projects into lumen and is negative for aneurysms and positive for stenosis. $R$ represents the radius of the artery, $R_{0}$ represents the radius of the normal artery, $L_{i}$ represents the length of the $i^{\text {th }}$ abnormal segment, $\alpha_{i}$ represents the distance from origin to the start of the $i^{\text {th }}$ abnormal segment and is given by

$$
\alpha_{i}=\sum_{j=1}^{i}\left(d_{j}+L_{j}\right)-L_{i}
$$

And $\beta_{i}$ represents the distance from the origin to the end of the $i^{\text {th }}$ abnormal segment and is given by

$$
\beta_{i}=\sum_{j=1}^{i}\left(d_{j}+L_{j}\right)
$$

where $d_{i}$ is the distance separating the start of $i^{\text {th }}$ abnormal segment from the end of the $(i-1)^{\text {th }}$ or from the start of the segment if $i=1$.

The constitutive equations of couple stress fluid are

$$
\begin{gathered}
\operatorname{div} \bar{q}=0 \\
T_{j i, j}=\rho \frac{\mathrm{d} u_{i}}{\mathrm{~d} t}, e_{i j k} T_{j k}^{A}+M_{j i, j}=0 \\
l_{i j}=-p \delta_{i j}+2 \mu_{i j} d_{i j}, \mu_{i j}=4 \eta \mu_{j, i}+4 \eta^{\prime} u_{i j}
\end{gathered}
$$

where $u_{i}$ is the velocity vector; $T_{i j}$ and $T_{i j}^{A}$ are the symmetric and antisymmetric parts of stress tensor $T_{i j}$ respectively; $M_{i j}$ is the couple stress tensor; $\mu_{i j}$ is the deviatoric part of $M_{i j}$ and $u_{i}$ is the vorticity vector; $d_{i j}$ is the symmetric part of the velocity gradient; $\eta$ and $\eta^{\prime}$ are constants associated with the couple stress; $p$ is the pressure and other terms have their usual meaning from tensor analysis.

With the assumptions of stenosis is to be mild (which implies that the variation of all the flow characteristics except pressure along the axial directions is negligible), the length of the tube is large compared to its radius that is $\frac{\delta}{R_{0}} \ll 1$, and introducing the following non-dimensional quantities.

$$
\begin{aligned}
& \bar{r}=\frac{r}{a}, \bar{z}=\frac{z}{L}, \bar{\delta}=\frac{\delta}{R_{0}}, \bar{p}=\frac{a^{2} p}{L \mu U} \\
& \bar{\alpha}=a \alpha=a \sqrt{\frac{\mu}{\eta}}, \bar{U}=\frac{u}{U} \\
& \bar{d}=\frac{d}{L}, \bar{L}=\frac{L}{L_{0}}
\end{aligned}
$$

where $U$ represents the average velocity.

The Equation (4) and Equation (5) becomes

$$
\begin{gathered}
\frac{\partial u}{\partial r}+\frac{u}{r}+\frac{\partial u}{\partial z}=0 \\
\frac{\partial p}{\partial r}=0
\end{gathered}
$$




$$
\frac{1}{r} \frac{\partial}{\partial r}\left\{r \frac{\partial}{\partial r}\left(1-\frac{1}{\bar{\alpha}^{2}} \nabla^{2}\right) u\right\}=\frac{\partial p}{\partial z}
$$

where $\nabla^{2}=\frac{1}{r} \frac{\partial}{\partial r}\left(r \frac{\partial}{\partial r}\right), u$ is the velocity $\bar{\alpha}^{2}=\frac{\mu}{\eta} a^{2}$ (couple stress parameter), since $\sqrt{\frac{\mu}{\eta}}$, a characteristic measure of polarity of the fluid model, has a dimension of length, $\bar{\alpha}$ indicates the ratio of the tube to material characteristic length $\sqrt{\frac{\eta}{\mu}}$, in the limit as $\eta \rightarrow 0$ i.e. $\bar{\alpha} \rightarrow \infty$, the Equation (9) reduces to Navier-Stokes equation.

The non-dimensional boundary conditions are

$$
\begin{gathered}
\frac{\partial u}{\partial r}=0 \text { at } r=0 \\
\frac{\partial^{2} u}{\partial r^{2}}-\frac{\eta}{r} \frac{\partial u}{\partial r}=0 \text { at } r=h(z) \\
\frac{\partial^{2} u}{\partial r^{2}}-\frac{\eta}{r} \frac{\partial u}{\partial r}=\text { is finite at } r=0 \\
\text { That implies } u \text { is finite at } r=0 \\
u=0 \text { at } r=h(z)
\end{gathered}
$$

\section{Solution}

Solving Equation (9) by using boundary conditions (10) to (14), we obtain the velocity as

$$
u=A I_{0}(\bar{\alpha} r)+\left(\frac{r^{2}}{4}+\frac{1}{\bar{\alpha}^{2}}\right) \frac{\mathrm{d} p}{\mathrm{~d} z}-\mathrm{d} \bar{\alpha}^{2}
$$

where

$$
\begin{gathered}
A=\frac{h\left(\frac{\eta-1}{2}\right) \frac{\mathrm{d} p}{\mathrm{~d} z}}{h \bar{\alpha}^{2} I_{0}(\bar{\alpha} h)-\bar{\alpha} I_{1}(\bar{\alpha} h)-\eta \bar{\alpha} I_{1}(\bar{\alpha} h)} \\
d=\frac{1}{\bar{\alpha}^{2}}\left[A I_{0}(\bar{\alpha} h)+\left(\frac{h^{2}}{4}+\frac{1}{\bar{\alpha}^{2}}\right) \frac{\mathrm{d} p}{\mathrm{~d} z}\right]
\end{gathered}
$$

where $I_{0}$ and $I_{1}$ are Modified Bessel functions of order zero and one respectively.

$$
\text { The dimensionless flux } q=\frac{q^{\prime}}{\pi a^{2} c} \text { is given by }
$$

Substituting Equation (15) in Equation (16) and then integrate we get

$$
\begin{aligned}
q & =\int_{0}^{h} 2 r\left\{\mathrm{AI}_{0}(\bar{\alpha} \mathrm{r})+\left(\frac{r^{2}}{4}+\frac{1}{\bar{\alpha}^{2}}\right) \frac{\mathrm{d} p}{\mathrm{~d} z}-\mathrm{d} \bar{\alpha}^{2}\right\} \mathrm{d} r \\
q & =\frac{2 A h}{\bar{\alpha}} I_{1}(\bar{\alpha} h)-h^{2} A I_{0}(\bar{\alpha} h)-\frac{h^{4}}{8} \frac{\mathrm{d} p}{\mathrm{~d} z}
\end{aligned}
$$


From Equation (17) we get $\frac{\mathrm{d} p}{\mathrm{~d} z}$ as

$$
\frac{\mathrm{d} p}{\mathrm{~d} z}=\frac{8 q}{h^{4} T}
$$

where

$$
\begin{gathered}
T=\frac{4(\eta-1)}{k h^{3}}\left[\frac{2 h}{\bar{\alpha}} I_{1}(\bar{\alpha} h)-h^{2} I_{0}(\bar{\alpha} h)\right]-1 \\
K=h \bar{\alpha}^{2} I_{0}(\bar{\alpha} h)-\bar{\alpha} I_{1}(\bar{\alpha} h)-\eta \bar{\alpha} I_{1}(\bar{\alpha} h)
\end{gathered}
$$

The pressure drop per wave length is given as $\Delta p=p(0)-p(\lambda)$ is

$$
\Delta p=-\int_{0}^{1} \frac{\mathrm{d} p}{\mathrm{~d} z} \mathrm{~d} z=-\int_{0}^{1} \frac{8 q}{h^{4} T} \mathrm{~d} z
$$

The resistance to the flow $\lambda$ is defined as

$$
\lambda=\frac{\Delta p}{q}=-\frac{1}{q} \int_{0}^{1} \frac{8 q}{h^{4} T} \mathrm{~d} z
$$

The pressure drop in the absence of stenosis $=1$ is denoted by $\Delta p_{n}$ and is obtained from Equation (19) as

$$
\Delta p_{n}=-\int_{0}^{1} \frac{8 q}{T} \mathrm{~d} z
$$

The resistance to the flow in the normal artery is denoted by $\lambda_{n}$ which is obtained from Equation (21) as

$$
\lambda_{n}=\frac{\Delta p_{n}}{q}=-\frac{1}{q} \int_{0}^{1} \frac{8 q}{T} \mathrm{~d} z
$$

The normalized resistance to the flow denoted by

$$
\bar{\lambda}=\frac{\lambda}{\lambda_{n}}
$$

The shear stress acting on the wall of tube is given by

$$
\tau_{w}=\left(-\mu \frac{\partial u}{\partial r}\right) \text { at } r=h(z)
$$

Introducing the dimensionless quantity

$$
\tau_{w}^{*}=\left(\frac{\tau_{w}}{\frac{\mu U}{d_{0}}}\right) \text { at } r=h(z)
$$

And using Equation (15) in Equation (24) we get (after dropping asterisks)

$$
\tau_{w}=-\mu\left[A \bar{\alpha} I_{1}(\bar{\alpha} h)+\left(\frac{h}{2}\right) \frac{8 q}{h^{4} T}\right]
$$

The shear stress at the wall in the absence of stenosis $=1$ denoted by $\left(\tau_{w}\right)_{n}$ is obtained 
from Equation (26) as

$$
\left(\tau_{w}\right)_{n}=-\mu\left[A \bar{\alpha} I_{1}(\bar{\alpha})+\left(\frac{1}{2}\right) \frac{8 q}{T}\right]
$$

The normalized shear stress at wall $\bar{\tau}_{w}$ is given by

$$
\bar{\tau}_{w}=\frac{\tau_{w}}{\left(\tau_{w}\right)_{n}}
$$

\section{Results and Analysis}

The expressions for Pressure drop $(\Delta p)$, resistance to the flow $(\bar{\lambda})$, and wall Shear stress $\left(\bar{\tau}_{w}\right)$ are given by Equation (19), Equation (23) and Equation (28) respectively and have been numerically evaluated by using MATHEMATICA Software for different values of corresponding parameters and presented graphically in the following Figures $2-14$.

Figure 2 to Figure 4 shows the effects of various parameters on resistance to the flow in a uniform tube with mild stenosis. It is observed that, the resistance to the flow increases with height and length of the stenosis but decreases with height and length of post stenotic dilatation.

It can be seen from the Figure 5 to Figure 8 that, the pressure drop increases with height of the stenosis and volumetric flow rate $(q)$ but decreases with couple stress

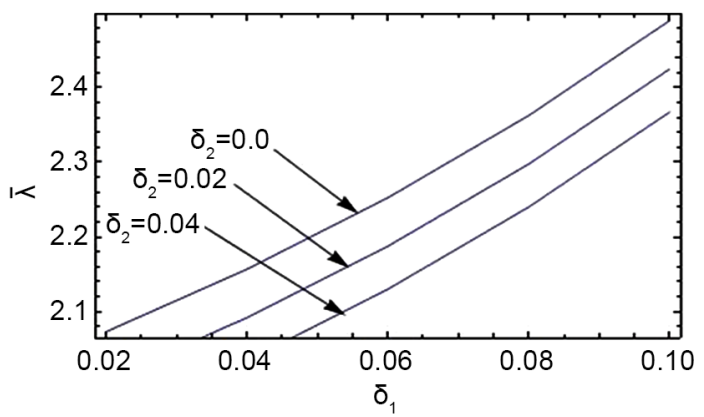

Figure 2. Variation of flow resistance $\bar{\lambda}$ with $\delta_{1}$ for different $\delta_{2}\left(d_{1}=d_{2}=0.2, L_{1}=L_{2}=0.2, r=\right.$ $0.8, q=0.1, \quad \bar{\alpha}=1, \eta=0.1, P=0.1)$.

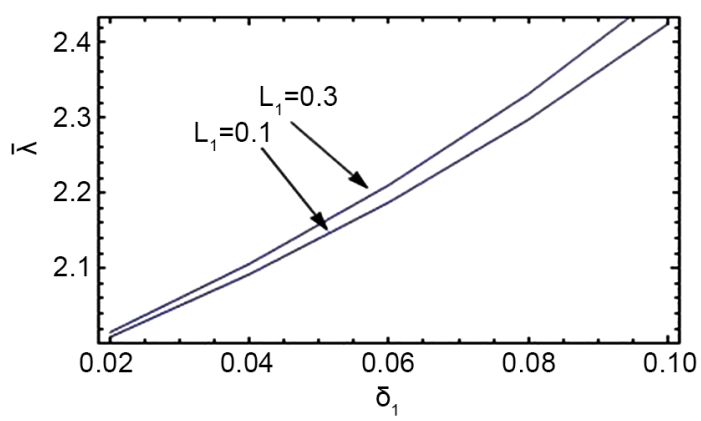

Figure 3. Variation of flow resistance $\bar{\lambda}$ with $\delta_{1}$ for different $L_{1}\left(d_{1}=d_{2}=0.2, L_{2}=0.2, r=0.8, q\right.$ $\left.=0.1, \quad \bar{\alpha}=1, \eta=0.1, P=0.1, \delta_{2}=0.02\right)$. 


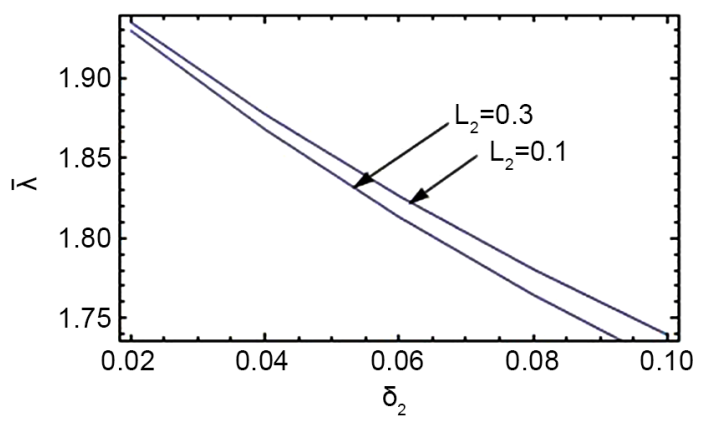

Figure 4. Variation of flow resistance $\bar{\lambda}$ with $\delta_{2}$ for different $L_{2}\left(d_{1}=d_{2}=0.2, L_{1}=0.1, r=0.8, q\right.$ $\left.=0.1, \quad \bar{\alpha}=1, \eta=0.1, P=0.1, \delta_{1}=0\right)$.

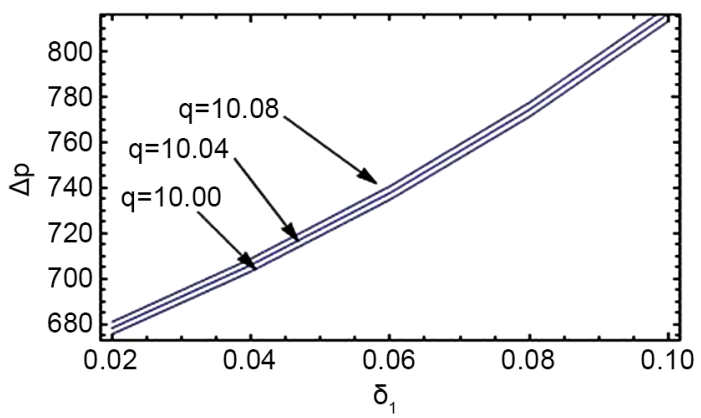

Figure 5. Variation of pressure drop $\Delta p$ with $\delta_{1}$ for different $q\left(d_{1}=d_{2}=0.2, L_{1}=L_{2}=0.2, r=0.8\right.$, $\left.\bar{\alpha}=1, \eta=0.1, P=0.1, \delta_{2}=0.01\right)$.

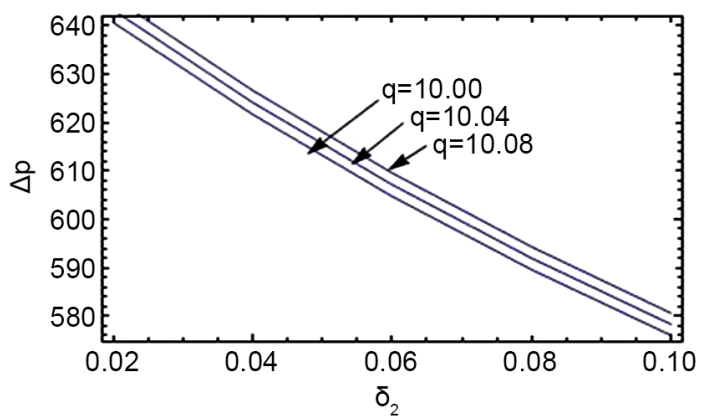

Figure 6. Variation of pressure drop $\Delta p$ with $\delta_{2}$ for different $q\left(d_{1}=d_{2}=0.2, L_{1}=L_{2}=0.2, r=0.8\right.$, $\left.\bar{\alpha}=1, \eta=0.1, P=0.1, \delta_{2}=0\right)$.

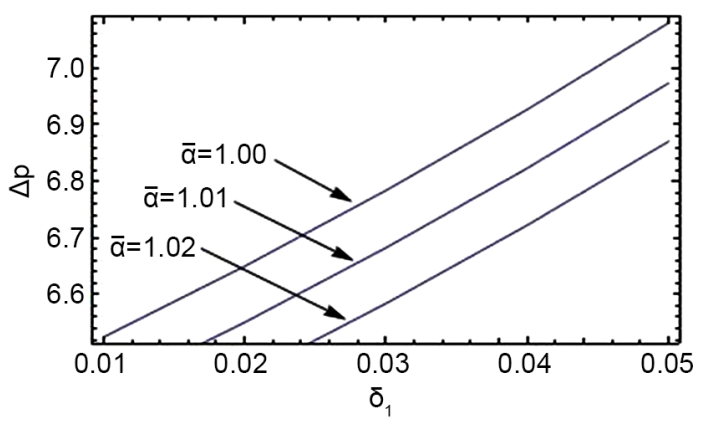

Figure 7. Variation of pressure drop $\Delta p$ with $\delta_{1}$ for different $\bar{\alpha} \quad\left(d_{1}=d_{2}=0.2, L_{1}=L_{2}=0.2, r=\right.$ $\left.0.8, q=0.1, \eta=0.1, P=0.1, \delta_{2}=0.01\right)$. 


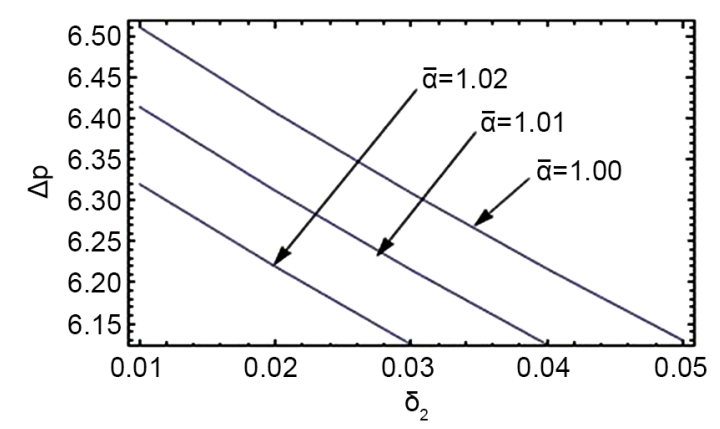

Figure 8. Variation of pressure drop $\Delta p$ with $\delta_{2}$ for different $\bar{\alpha} \quad\left(d_{1}=d_{2}=0.2, L_{1}=L_{2}=0.2, r=\right.$ $\left.0.8, q=0.1, \eta=0.1, P=0.1, \delta_{1}=0\right)$.

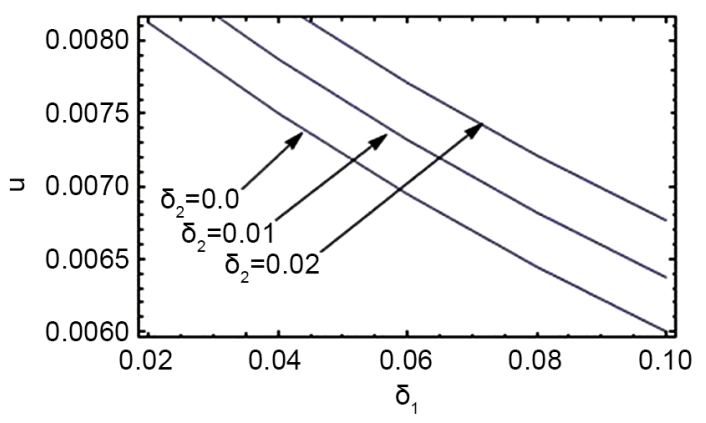

Figure 9. Variation of velocity $u$ with $\delta_{1}$ for different $\delta_{2}\left(d_{1}=d_{2}=0.2, L_{1}=L_{2}=0.2, r=0.8, q=\right.$ $0.1, \quad \bar{\alpha}=1, \eta=1.7, P=0.1$.

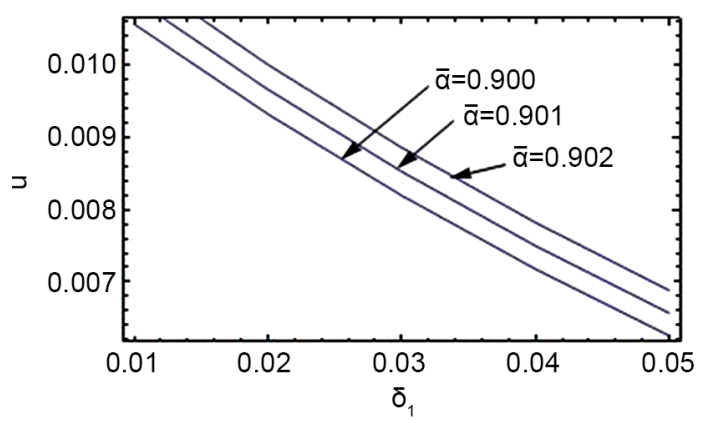

Figure 10. Variation of velocity $u$ with $\delta_{1}$ for different $\bar{\alpha} \quad\left(d_{1}=d_{2}=0.2, L_{1}=L_{2}=0.2, r=0.8, q=\right.$ $\left.0.1, \bar{\alpha}=1, \eta=1.3, P=0.1, \delta_{2}=0\right)$.

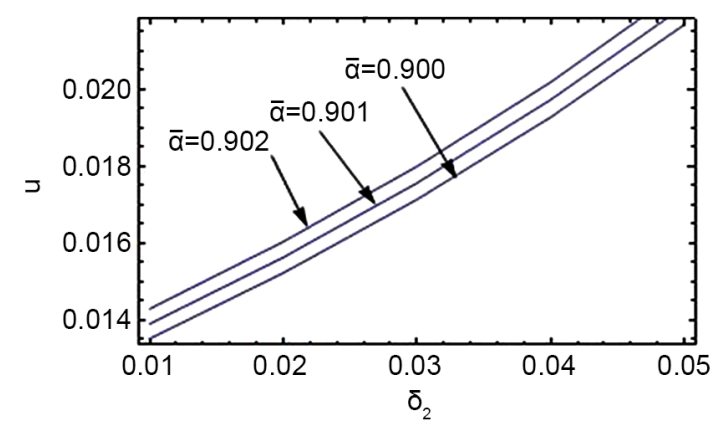

Figure 11. Variation of velocity $u$ with $\delta_{2}$ for different $\bar{\alpha} \quad\left(d_{1}=d_{2}=0.2, L_{1}=L_{2}=0.2, r=0.8, q=\right.$ $\left.0.1, \quad \bar{\alpha}=1, \eta=1.3, P=0.1, \delta_{1}=0\right)$. 


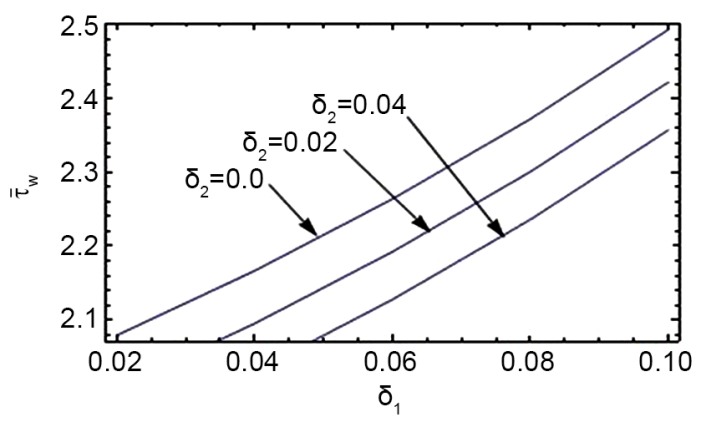

Figure 12. Variation of Shear stress $\bar{\tau}_{w}$ with $\delta_{1}$ for different $\delta_{2}\left(d_{1}=d_{2}=0.2, L_{1}=L_{2}=0.2, \delta_{2}=0\right.$, $r=0.8, \mu=0.2, q=0.1, \quad \bar{\alpha}=1, \eta=1.8)$.

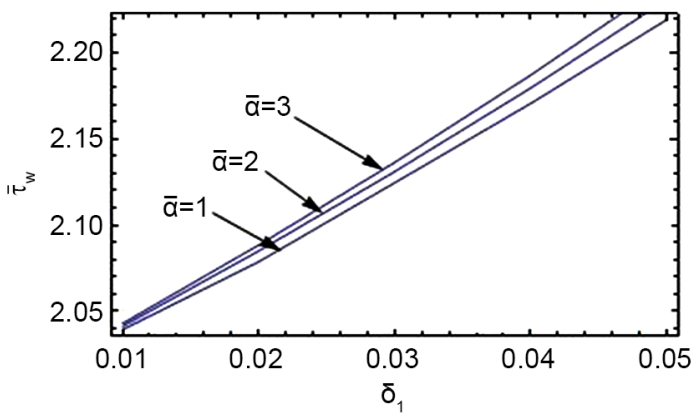

Figure 13. Variation of Shear stress $\bar{\tau}_{w}$ with $\delta_{1}$ for different $\bar{\alpha} \quad\left(d_{1}=d_{2}=0.2, L_{1}=L_{2}=0.2, \delta_{2}=\right.$ $0, r=0.8, \mu=0.2, q=0.1, \eta=5)$.

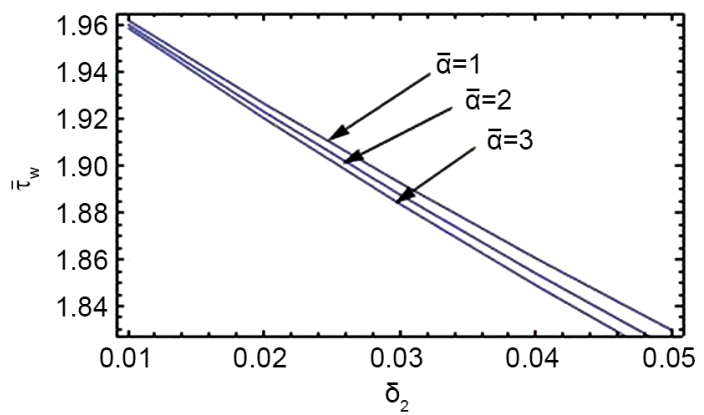

Figure 14. Variation of Shear stress $\bar{\tau}_{w}$ with $\delta_{2}$ for different $\bar{\alpha} \quad\left(d_{1}=d_{2}=0.2, L_{1}=L_{2}=0.2, \delta_{1}=\right.$ $0, r=0.8, \mu=0.2, q=0.1, \eta=5)$.

fluid parameter $(\bar{\alpha})$. It can also be observed that, the pressure drop decreases with height of post stenotic dilatation $\left(\delta_{2}\right)$ and couple stress fluid parameter $(\bar{\alpha})$ but increases with volumetric flow rate $(q)$.

It is also noticed that velocity is decreases with height of the stenosis and increases with couple stress fluid parameter $\bar{\alpha}$ (Figure 9 \& Figure 10). And velocity is increases with height of the post stenotic dilatation and couple stress fluid parameter (Figure 10 \& Figure 11).

It is identified from the Figure 12 and Figure 13 that, the wall shear stress increases with height of the stenosis and couple stress fluid parameter but decreases with height of the post stenotic dilatation and couple stress fluid parameter (Figure 14). 


\section{Conclusion}

A mathematical model for stenosis and post stenotic dilatation on the steady flow of couple stress fluid in a uniform tube has been presented. Solutions have been obtained for mild stenosis. It has been obtained that the resistance to the flow, pressure drop and shear stress increase with the height and length of the stenosis but decrease in the case of post stenotic dilatation. However, wall shear stress increases with height of the stenosis and couple stress parameter but decreases with post stenotic dilatation and couple stress fluid parameter.

\section{References}

[1] Shukla, J.B., Parihar, R.S. and Rao, B.R.P. (1980) Effects of Stenosis on Non-Newtonian Flow of the Blood in an Artery. Bulletin of Mathematical Biology, 42, 283-294. https://doi.org/10.1007/BF02460787

[2] Chakravarty, S. and Datta, A. (1990) Dynamic Response of Arterial Blood Flow in the Presence of Multi Stenosis. Mathematical and Computer Modelling, 13, 37-55.

https://doi.org/10.1016/0895-7177(90)90062-R

[3] Philip, D. and Chandra, P. (1996) Flow of Eringen Fluid (Simple Miofluid) through an Artery with Mild Stenosis. International Journal of Engineering Science, 34, 879. https://doi.org/10.1016/0020-7225(95)00077-1

[4] Mandal, P.K. (2005) An Unsteady Analysis of Non-Newtonian Blood Flow through Tapered Arteries with a Stenosis. International Journal of Non-Linear Mechanics, 40, 151-164. https://doi.org/10.1016/j.ijnonlinmec.2004.07.007

[5] Sankar, D.S. and Hemalatha, K. (2007) A Non-Newtonian Fluid Flow Model for Blood Flow through a Catheterized Artery-Steady Flow. Applied Mathematical Modelling, 31, 1847-1864. https://doi.org/10.1016/j.apm.2006.06.009

[6] Prasad, K.M. and Radhakrishnamacharya, G. (2008) Flow of Herschel-Bulkley Fluid through an Inclined Tube of Non-Uniform Cross Section with Multiple Stenosis. Archives of Mechanics, 60, 161-172.

[7] Saleh, M.M.M. and Khan, M.Y.A. (2011) Effect of Paired Stenosis on Blood Flow through Small Artery. Journal of Mathematics Research, 3, 224-229. https://doi.org/10.5539/jmr.v3n2p224

[8] Sreenadh, S., Raga Pallavi, A. and Satyanarayana, Bh. (2011) Flow of a Casson Fluid through an Inclined Tube of Non-Uniform Cross Section with Multiple Stenoses. Advances in Applied Science Research, 2, 340-349.

[9] Bali, R. and Awasthi, U. (2012) A Casson Fluid Model for Multiple Stenosed Artery in the Presence of Magnetic Field. Applied Mathematics, 3, 436-441.

https://doi.org/10.4236/am.2012.35066

[10] Venkatesan, J., Sankar, D.S., Hemalatha, K. and Yatim, Y. (2013) Mathematical Analysis of Casson Fluid Model for Blood Rheology in Stenosed Narrow Arteries. Journal of Applied Mathematics, 2013, Article ID: 583809, 11 p.

[11] Stokes, V.K. (1966) Couple Stresses in Fluids. Physics of Fluids, 9, 1709-1715. https://doi.org/10.1063/1.1761925

[12] Srivastava, V.P. (2003) Flow of a Couple Stress Fluid Representing Blood through Stenotic Vessels with a Peripheral Layer. Indian Journal of Pure and Applied Mathematics, 34, 17241740 . 
[13] Srinivasacharya, D. and Srikanth, D. (2008) Effect of Couple Stresses on the Flow in a Constricted Annulus. Archive of Applied Mechanics, 78, 251-257.

https://doi.org/10.1007/s00419-007-0157-6

[14] Maruthi Prasad, K. and Radhakrishnamacharya, G. (2008) Effect of Peripheral Layer on Peristaltic Transport of a Couple Stress Fluid. International Journal of Fluid Mechanics Research, 36, 573-583.

[15] Rathod, V.P. and Tanveer, S. (2009) Pulsatile Flow of Couple Stress Fluid through a Porous Medium with Periodic Body Acceleration and Magnetic Field. Bulletin of the Malaysian Mathematical Sciences Society, 32, 245-259.

[16] Srikanth, D. and Kebede, T. (2012) Mathematical Analysis of non-Newtonian Fluid Flow through Multiple Stenotic Artery in the Presence of Catheter-A Pulsatile Flow. International Journal of Nonlinear Science, 13, 15-27.

[17] Maiti, S. and Misra, J.C. (2012) Peristaltic Transport of a Couple Stress Fluid Some Applications to Hemodynamics. arXiv:1006.0177 [physics.flu-dyn]

[18] Gurju, A. and Radhakrishnamacharya, G. (2013) Effect of Slip Condition on Couple Stress Fluid Flow through Porous Medium with Stenosis. International Journal of Scientific \& Engineering Research, 4, 28-33.

[19] Pincombe, B. and Mazumdar, J. (1997) The Effects of Post- Stenotic Dilatations on the Flow of a Blood Alogue through Stenosed Coronary Arteries. Mathematical and Computer Modelling, 25, 57-70. https://doi.org/10.1016/S0895-7177(97)00039-3

[20] Singh, A.K. and Singh, D.P. (2012) A Computational Study of Bingham Plastic Flow of Blood through an Artery by Multiple Stenosis and Post Dilatation. Advances in Applied Science Research, 3, 3285-3290.

[21] Sanjeev, K. and Chandrashekhar, D. (2013) Blood Flow Resistance for a Small Artery with the Effect of Multiple Stenosis and Post Stenotic Dilatation. International Journal of Engineering Sciences and Emerging Technologies, 6, 57-64.

[22] Maruthi Prasad, K., Bhuvana Vijaya, R. and Umadevi, C. (2015) Effects of Stenosis and Post Stenotic Dilatations on Jeffrey Fluid Flow in Arteries. International Journal of Research in Engineering and Technology, 4, 195-201. https://doi.org/10.15623/ijret.2015.0413032

\section{Submit or recommend next manuscript to SCIRP and we will provide best service for you:}

Accepting pre-submission inquiries through Email, Facebook, LinkedIn, Twitter, etc.

A wide selection of journals (inclusive of 9 subjects, more than 200 journals)

Providing 24-hour high-quality service

User-friendly online submission system

Fair and swift peer-review system

Efficient typesetting and proofreading procedure

Display of the result of downloads and visits, as well as the number of cited articles

Maximum dissemination of your research work

Submit your manuscript at: http://papersubmission.scirp.org/

Or contact ajcm@scirp.org 